\title{
There are no topologically transitive operators in the noncommutative Schwartz space
}

\author{
Krzysztof PiszczeK(D) AND AdAm Przestacki
}

\begin{abstract}
The aim of this note is to prove that there are no topologically transitive operators in the noncommutative Schwartz space.
\end{abstract}

Mathematics Subject Classification. Primary 47A16; Secondary 47B37, 46A 45, 46H30, 46H35.

Keywords. Space of rapidly decreasing/slowly increasing sequences, Hypercyclic operator, Spectrum, Holomorphic functional calculus.

1. Introduction. Let $s$ and $s^{\prime}$ be the spaces of rapidly decreasing and slowly increasing sequences, respectively, equipped with their natural locally convex topologies. The so-called noncommutative Schwartz space is the space $\mathcal{S}:=L\left(s^{\prime}, s\right)$ of all bounded linear operators acting from $s^{\prime}$ into $s$. This space becomes a Fréchet algebra in a natural way: if $T_{1}$ and $T_{2}$ are in $\mathcal{S}$, then the product $T_{1} T_{2}$ is defined by the formula $T_{1} T_{2}=T_{1} \circ \iota \circ T_{2}$, where $\iota$ is the natural embedding of $s$ into $s^{\prime}$. In fact, $\mathcal{S}$ embeds algebraically into the $C^{*}$-algebra $\mathcal{B}\left(\ell_{2}\right)$ of all bounded and linear operators on the Hilbert space $\ell_{2}$.

The noncommutative Schwartz space is isomorphic (as a Fréchet *-algebra) to a number of other natural objects of analysis, e.g., $\mathcal{S} \simeq \mathcal{S}\left(\mathbb{R}^{2}\right)$ - the Schwartz space of rapidly decreasing functions on $\mathbb{R}^{2}$ equipped with the Volterra multiplication $(f \cdot g)(x, y):=\int_{\mathbb{R}} f(x, z) g(z, y) d z$ and involution $f^{*}(x, y):=\overline{f(y, x)}$. It plays an important role, e.g., in K-theory - see [4,12], cyclic cohomology for crossed products - see $[9,14]$, noncommutative geometry - see [3], operator spaces - see $[7,8]$. Another motivation comes from quantum mechanics where $\mathcal{S}$ is called the space of physical states and its dual is the so-called space of observables - see [6] for details.

Since $\mathcal{S}$ is a Fréchet algebra of operators, it is natural to ask about the dynamical properties of the elements of $\mathcal{S}$. Recall that an operator $T: s^{\prime} \rightarrow s$ is topologically transitive if for every two non-empty and open sets $U \subset s^{\prime}$, 
$V \subset s$, there exists $n \geq 0$ such that $T^{n}(U) \cap V \neq \emptyset$ and hypercyclic if there exists $x \in s^{\prime}$ such that the set $\left\{T^{n} x: n \in \mathbb{N}\right\}$ is dense in $s$. It is clear that hypercyclicity of $T$ implies that it is topologically transitive. Formally it could happen that the latter is a weaker property since $s^{\prime}$ is not a metric space.

The main goal of this note is to show that there are no topologically transitive operators in $\mathcal{S}$. The main difficulty of the paper is to understand the spectral properties of operators from $\mathcal{S}$, those are investigated in Sect. 2.

We refer the reader to $[1,10,11]$ for unexplained details from linear dynamics and functional analysis, respectively.

2. Notation and terminology. Recall that

$$
s=\left\{\xi=\left(\xi_{j}\right)_{j \in \mathbb{N}} \subset \mathbb{C}^{\mathbb{N}}:|\xi|_{t}^{2}:=\sum_{j=1}^{+\infty}\left|\xi_{j}\right|^{2} j^{2 t}<+\infty \text { for all } t \geqslant 0\right\}
$$

and its topological dual

$$
s^{\prime}=\left\{\eta=\left(\eta_{j}\right)_{j \in \mathbb{N}} \subset \mathbb{C}^{\mathbb{N}}:\left(|\eta|_{t}^{\prime}\right)^{2}:=\sum_{j=1}^{+\infty}\left|\eta_{j}\right|^{2} j^{-2 t}<+\infty \text { for some } t \geqslant 0\right\}
$$

are the so-called spaces of rapidly decreasing and slowly increasing sequences, respectively.

Furthermore we consider the space $\mathcal{S}:=L\left(s^{\prime}, s\right)$ of all linear and continuous operators from $s^{\prime}$ into $s$, equipped with the topology of uniform convergence on bounded sets. Consequently, the topology of $\mathcal{S}$ is given by the scale $\left(\|\cdot\|_{t}\right)_{t \geqslant 0}$ of norms, defined as

$$
\|T\|_{t}:=\sup \left\{|T \eta|_{t}:|\eta|_{t}^{\prime} \leqslant 1\right\} \quad(T \in \mathcal{S}, t \geqslant 0) .
$$

If we denote $H_{t}:=\ell_{2}\left(\left(j^{t}\right)_{j \in \mathbb{N}}\right), t \in \mathbb{R}$, then $H_{t}^{\prime} \cong H_{-t}$ and every $T \in \mathcal{S}$ is a Hilbert space operator in the sense that $T: H_{t}^{\prime} \rightarrow H_{t}$ and

$$
\|T\|_{t}=\|T\|_{H_{t}^{\prime} \rightarrow H_{t}} \quad(t \geqslant 0) .
$$

In other words, if we denote by $D_{t}:=\operatorname{diag}\left(j^{t}\right), t \in \mathbb{R}$, an infinite diagonal matrix, then $D_{t}$ becomes simultaneously an isometry $D_{t}: H_{t} \rightarrow \ell_{2}$ and $D_{t}: \ell_{2} \rightarrow H_{t}^{\prime}$ and

$$
\|T\|_{t}=\left\|D_{t} T D_{t}\right\|_{\mathcal{B}\left(\ell_{2}\right)} \quad(T \in \mathcal{S}, t \geqslant 0) .
$$

In particular, $\mathcal{S}=\operatorname{proj}_{t \geqslant 0} \mathcal{B}\left(H_{t}^{\prime}, H_{t}\right)=\operatorname{proj}_{k \in \mathbb{N}} \mathcal{B}\left(H_{k}^{\prime}, H_{k}\right)$. We will be using these properties interchangably.

Since $s \hookrightarrow s^{\prime}$, we can define multiplication in $\mathcal{S}$ as

$$
T_{1} T_{2}:=T_{1} \circ \iota \circ T_{2} \quad\left(T_{1}, T_{2} \in \mathcal{S}\right),
$$

where $\iota: s \rightarrow s^{\prime}, \iota(\xi):=\xi$ is the formal embedding. Altogether it turns $\mathcal{S}$ into an $m$-convex Fréchet algebra. It comes endowed also with the involution (or the adjoint map) given as

$$
\left\langle T^{*} \xi, \eta\right\rangle:=\langle\xi, T \eta\rangle \quad\left(\xi, \eta \in s^{\prime}, T \in \mathcal{S}\right) .
$$

It is worth noting that $s \hookrightarrow \ell_{2}$ and, by dualization, also $\ell_{2} \hookrightarrow s^{\prime}$ therefore $\mathcal{S}$ is algebraically contained in the $C^{*}$-algebra $\mathcal{B}\left(\ell_{2}\right)$ of all bounded and linear 
operators on the Hilbert space $\ell_{2}$. Therefore multiplication in $\mathcal{S}$ is essentially the multiplication in $\mathcal{B}\left(\ell_{2}\right)$ with the additional property that the resulting operator belongs to $\mathcal{S}$. The same applies to involution.

The unitization of $\mathcal{S}$ will be denoted by $\mathcal{S}_{1}$. Clearly, the unit in $\mathcal{S}_{1}$ is the identity operator on $\ell_{2}$ denoted by $\mathbb{1}$. The algebra $\mathcal{S}$ is called the noncommutative Schwartz space and the elements of $\mathcal{S}$ are called smooth operators. We refer the reader to $[2,13]$ for more information on the properties of this algebra.

3. Spectral properties of operators in $\mathcal{S}$. We start by showing some spectral properties of smooth operators.

Proposition 3.1 ([5, Proposition 3.1 and Theorem 3.3]). Every smooth operator is compact, i.e., $\mathcal{S} \hookrightarrow \mathcal{K}\left(\ell_{2}\right)$ and

$$
\sigma_{\mathcal{S}_{1}}(T)=\sigma_{\mathcal{B}\left(\ell_{2}\right)}(T) \quad(T \in \mathcal{S}) .
$$

In particular, the spectrum of every smooth operator consists of zero and a (possibly) null sequence of eigenvalues.

Lemma 3.2. For any $t \in \mathbb{R}$ and every smooth operator $T \in \mathcal{S}$, we have

$$
\sigma_{\mathcal{B}\left(\ell_{2}\right)}\left(D_{t} T D_{-t}\right) \subset \sigma_{\mathcal{S}_{1}}(T) .
$$

Proof. Let $t \in \mathbb{R}$ and $T \in \mathcal{S}$ be fixed. Suppose that $\lambda \in \rho_{\mathcal{S}_{1}}(T)$, i.e., there is $S \in \mathcal{S}$ such that

$$
\left(S-\frac{1}{\lambda} \mathbb{1}\right)(T-\lambda \mathbb{1})=(T-\lambda \mathbb{1})\left(S-\frac{1}{\lambda} \mathbb{1}\right)=\mathbb{1},
$$

where $\mathbb{1}$ is the identity operator on $\ell_{2}$. Then

$$
\ell_{2} \stackrel{D_{-t}}{\longrightarrow} H_{t} \hookrightarrow s^{\prime} \stackrel{S}{\longrightarrow} s \hookrightarrow H_{t} \stackrel{D_{t}}{\longrightarrow} \ell_{2}
$$

and

$$
\left(D_{t} S D_{-t}-\frac{1}{\lambda} \mathbb{1}\right)\left(D_{t} T D_{-t}-\lambda \mathbb{1}\right)=\left(D_{t} T D_{-t}-\lambda \mathbb{1}\right)\left(D_{t} S D_{-t}-\frac{1}{\lambda} \mathbb{1}\right)=\mathbb{1}
$$

Consequently, $\lambda \in \rho_{\mathcal{B}\left(\ell_{2}\right)}\left(D_{t} T D_{-t}\right)$ and the proof is thereby complete.

Corollary 3.3. If a smooth operator $T \in \mathcal{S}$ satisfies

$$
\sigma_{\mathcal{S}_{1}}(T) \subset \mathbb{D}
$$

then the sequence $\left(T^{n}\right)_{n \in \mathbb{N}}$ is bounded in $\mathcal{S}$.

Proof. Let the smooth operator $T \in \mathcal{S}$ satisfy (2). Since $D_{t}^{-1}=D_{-t}$, we obtain that for every $n \in \mathbb{N}$ and every $t \geqslant 0$,

$$
\begin{aligned}
\left\|T^{n}\right\|_{t} & =\left\|D_{t} T^{n} D_{t}\right\|_{\mathcal{B}\left(\ell_{2}\right)} \\
& =\|\underbrace{D_{t} T D_{-t} D_{t} T D_{-t} \cdots D_{t} T D_{-t}}_{n-1} D_{t} T D_{t}\|_{\mathcal{B}\left(\ell_{2}\right)} \\
& \leqslant\left\|\left(D_{t} T D_{-t}\right)^{n-1}\right\|_{\mathcal{B}\left(\ell_{2}\right)}\|T\|_{t} .
\end{aligned}
$$


From Lemma 3.2, the spectral radius formula, and compactness of the spectrum, it now follows that there is $\varepsilon>0$ such that

$$
\nu\left(D_{t} T D_{-t}\right)=\lim _{n \rightarrow \infty}\left\|\left(D_{t} T D_{-t}\right)^{n}\right\|_{\mathcal{B}\left(\ell_{2}\right)}^{1 / n} \leqslant 1-\varepsilon .
$$

Hence there is $N \in \mathbb{N}$ such that for every $n \geqslant N$, we have

$$
\left\|\left(D_{t} T D_{-t}\right)^{n}\right\|_{\mathcal{B}\left(\ell_{2}\right)} \leqslant 1 \text {. }
$$

If we now define $C_{t}:=\max \left\{\|T\|_{t},\left\|T^{2}\right\|_{t}, \ldots,\left\|T^{N}\right\|_{t}, 1\right\} \cdot\|T\|_{t}$, then

$$
\sup _{n \in \mathbb{N}}\left\|T^{n}\right\|_{t} \leqslant C_{t}<\infty
$$

Consequently, $\left(T^{n}\right)_{n \in \mathbb{N}}$ is a bounded sequence in the noncommutative Schwartz space.

\section{Main result.}

Theorem 3.1. There are no topologically transitive operators in $\mathcal{S}$. In particular, the operators in $\mathcal{S}$ are not hypercyclic.

Proof. Let $T \in \mathcal{S}$ be arbitrary. There are two possible cases: either $\sigma_{\mathcal{S}_{1}}(T)=$ $\{0\}$ or there exists $0 \neq \lambda \in \sigma_{\mathcal{S}_{1}}(T)$.

If $\sigma_{\mathcal{S}_{1}}(T)=\{0\}$, then from Corollary 3.3 , the sequence $\left(T^{n}\right)_{n \in \mathbb{N}}$ is bounded in $\mathcal{S}$ and therefore it is equicontinuous. In particular, for every zero neighbourhood $U \subset s^{\prime}$, there is a zero neighbourhood $V \subset s$ such that

$$
T^{n}(U) \subset \frac{1}{2} V \quad(n \in \mathbb{N})
$$

We choose now $\xi \in s \backslash V$ and suppose that there is $n \in \mathbb{N}$ and $\eta \in s$ such that

$$
\eta \in T^{n}(U) \cap\left(\xi+\frac{1}{2} V\right) .
$$

This implies that for some $\zeta \in \frac{1}{2} V$, we have

$$
\xi=\eta-\zeta \in \frac{1}{2} V+\frac{1}{2} V=V .
$$

This contradicts the choice of $\xi \in s$ and shows that in this case $T$ is not topologically transitive.

If there exists $0 \neq \lambda \in \sigma_{\mathcal{S}_{1}}(T)$, then from Proposition 3.1, it follows that $\lambda$ is an eigenvalue and we let $f$ be a holomorphic function on a neighbourhood of $\sigma_{\mathcal{S}_{1}}(T)$ such that $f(\lambda)=1$ and $f(z)=0$ for $z \in \sigma_{\mathcal{S}_{1}}(T) \backslash\{\lambda\}$. Using the holomorphic functional calculus (which is available in $\mathcal{S}$ by [12, Lemma 1.3]), we can now consider the operator $f(T) \in \mathcal{S}$. Let $M=\operatorname{Im}(f(T))$. It is clear that $M$ is a non-trivial and finite dimensional subspace of $s$ (every non-zero element of $M$ is an eigenvector of the compact operator $f(T)$ ). The properties of the functional calculus imply that the diagram

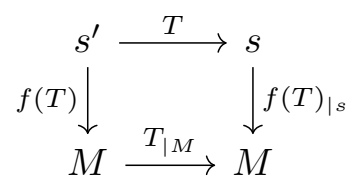


commutes and one can easily verify that topological transitivity of $T$ would imply topological transitivity of $T_{\mid M}$. Since $M$ is finite dimensional this implies that $T$ is not topologically transitive.

Acknowledgements. The research of both authors was partially supported by the National Center of Science, Poland, grant no. UMO-2013/10/A/ST1/00091.

Open Access. This article is licensed under a Creative Commons Attribution 4.0 International License, which permits use, sharing, adaptation, distribution and reproduction in any medium or format, as long as you give appropriate credit to the original author(s) and the source, provide a link to the Creative Commons licence, and indicate if changes were made. The images or other third party material in this article are included in the article's Creative Commons licence, unless indicated otherwise in a credit line to the material. If material is not included in the article's Creative Commons licence and your intended use is not permitted by statutory regulation or exceeds the permitted use, you will need to obtain permission directly from the copyright holder. To view a copy of this licence, visit http://creativecommons. org/licenses/by/4.0/.

Publisher's Note Springer Nature remains neutral with regard to jurisdictional claims in published maps and institutional affiliations.

\section{References}

[1] Bayart, F., Matheron, É.: Dynamics of Linear Operators: Cambridge Tracts in Mathematics, vol. 179. Cambridge University Press, Cambridge (2009)

[2] Ciaś, T.: On the algebra of smooth operators. Studia Math. 218(2), 145-166 (2013)

[3] Connes, A.: Noncommutative Geometry. Academic Press Inc, San Diego (1994)

[4] Cuntz, J.: Cyclic theory and the bivariant Chern-Connes character. In: Noncommutative Geometry, Lecture Notes in Mathematics, vol. 1831, pp. 73-135. Springer, Berlin (2004)

[5] Domański, P.: Algebra of smooth operators (unpublished note)

[6] Dubin, D.A., Hennings, M.A.: Quantum Mechanics, Algebras and Distributions. Pitman Research Notes in Mathematics Series, vol. 238, Longman Scientific \& Technical, Harlow (copublished in the United States with John Wiley \& Sons, Inc., New York) (1990)

[7] Effros, E.G., Webster, C.: Operator analogues of locally convex spaces. In: Operator Algebras and Applications (Samos, 1996), pp. 163-207. NATO Adv. Sci. Inst. Ser. C Math. Phys. Sci., vol. 495. Kluwer Acad. Publ., Dordrecht (1997)

[8] Effros, E.G., Winkler, S.: Matrix convexity: operator analogues of the bipolar and Hahn-Banach theorems. J. Funct. Anal. 144(1), 117-152 (1997)

[9] Elliott, G.A., Natsume, T., Nest, R.: Cyclic cohomology for one-parameter smooth crossed products. Acta Math. 160(3-4), 285-305 (1988)

[10] Grosse-Erdmann, K.-G., Peris Manguillot, A.: Linear Chaos. Universitext, Springer, London (2011) 
[11] Meise, R.G., Vogt, D.: Introduction to Functional Analysis. Translated from the German by M. S. Ramanujan and revised by the authors. Oxford Graduate Texts in Mathematics, vol. 2. The Clarendon Press, Oxford University Press, New York (1997)

[12] Phillips, N.C.: K-theory for Fréchet algebras. Int. J. Math. 2(1), 77-129 (1991)

[13] Piszczek, K.: Automatic continuity and amenability in the non-commutative Schwartz space. J. Math. Anal. Appl. 432(2), 954-964 (2015)

[14] Schweitzer, L.B.: Spectral invariance of dense subalgebras of operator algebras. Int. J. Math. 4(2), 289-317 (1993)

Krzysztof Piszczek And Adam Przestacki

Faculty of Mathematics and Computer Science

A. Mickiewicz University in Poznań

Uniwersytetu Poznańskiego 4

61-614 Poznań

Poland

e-mail: kpk@amu.edu.pl

Adam Przestacki

e-mail: adamp@amu.edu.pl

Received: 6 August 2021

Accepted: 7 January 2022. 